\title{
Neural Dynamics and Gamma Oscillation on a Hybrid Excitatory-Inhibitory Complex Network (Student Abstract)
}

\author{
Yuan Wang, ${ }^{1}$ Xia Shi, ${ }^{2 *}$ Bo Cheng, ${ }^{1}$ Junliang Chen ${ }^{1}$ \\ ${ }^{1}$ State Key Laboratory of Networking and Switching Technology, ${ }^{2}$ School of Science, \\ Beijing University of Posts and Telecommunications, Beijing 100876, P.R. China \\ \{yuanwang, chengbo, chjl\}@bupt.edu.cn, shixiabupt@163.com
}

\begin{abstract}
This paper investigates the neural dynamics and gamma oscillation on a complex network with excitatory and inhibitory neurons (E-I network), as such network is ubiquitous in the brain. The system consists of a small-world network of neurons, which are emulated by Izhikevich model. Moreover, mixed Regular Spiking (RS) and Chattering $(\mathrm{CH})$ neurons are considered to imitate excitatory neurons, and Fast Spiking (FS) neurons are used to mimic inhibitory neurons. Besides, the relationship between synchronization and gamma rhythm is explored by adjusting the critical parameters of our model. Experiments visually demonstrate that the gamma oscillations are generated by synchronous behaviors of our neural network. We also discover that the Chattering $(\mathrm{CH})$ excitatory neurons can make the system easier to synchronize.
\end{abstract}

\section{Introduction}

In recent years, artificial intelligence techniques directed by neuroscience have attempted to closely mimic the cerebral cortex, based on research of rich spatiotemporal neural dynamics, different spiking and bursting patterns and various learning and memory rules (Hassabis et al. 2017). In particular, Spiking Neural Networks (SNNs) are considered as a typical biologically inspired method based on the spatiotemporal dynamics of neural networks to mimic the information transfer processing.

However, it is challenging for SNNs to efficiently perform and accurately capture the biological properties, since most such SNNs algorithms depend on the different variants of Integrate-and-Fire (IF) neuron model which produces less biophysical properties of neural networks (Wu et al. 2019).

The gamma rhythm occurs through synchronization between various spiking or bursting in neural circuits. Neural synchronization also plays a key role when transferring and coding mechanisms in different regions of the cerebral cortex. Therefore, it is of great importance to investigate the processing of synchronization and gamma oscillation in the brain. Learning more informative context of how mammalian neocortex mechanism is performing in information

\footnotetext{
* Xia Shi is the corresponding author. Copyright (C) 2020, Association for the Advancement of Artificial Intelligence (www.aaai.org). All rights reserved.
}

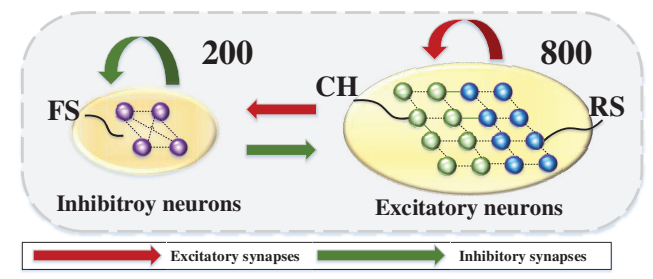

Figure 1: (Color online) Schematic presentation of the proposed network architecture of excitatory and inhibitory neurons.

processing and artificial intelligence is particularly important.

In this work, the Izhikevich neuron model (Izhikevich 2003 ) is used to explore the gamma rhythm and the synchronization transition of cortical neural network with both types of excitatory and inhibitory neurons. In particular, excitatory neurons are composed of hybrid Regular Spiking (RS) and Chattering $(\mathrm{CH})$ types neurons. We also focus on how the synaptic coupling and the network properties would affect the rhythm types, the synchronous states, and the relationship between synchronization and the rhythm transition.

\section{Methods}

The Izhikevich model, as a core element, is used to simulate the individual dynamics of each neuron in an excitatoryinhibitory balanced cortical neural network.

It can be expressed by a system of ordinary differential equations as below:

$$
\begin{gathered}
\frac{d v_{i}}{d t}=0.04 v_{i}^{2}+5 v_{i}+140-u_{i}+I_{i}^{e x t}+I_{i}^{s y n}+D \xi_{i} \\
\frac{d u_{i}}{d t}=a_{i}\left(b_{i} v_{i}-u_{i}\right) \\
\text { if } \quad v_{i} \geq V_{\text {peak }}(+30 \mathrm{mV}), \quad\left\{\begin{array}{l}
v_{i} \leftarrow c_{i}, \\
u_{i} \leftarrow u_{i}+d_{i} .
\end{array}\right. \\
I_{i}^{\text {syn }}=\sum_{j=1, j \neq i}^{N} g_{i j} w_{i j}\left(v_{j}-v_{i}\right)
\end{gathered}
$$


Table 1: Parameter values used in our computations.

\begin{tabular}{lcccc}
\hline \multirow{2}{*}{ Types of neuron } & \multicolumn{4}{c}{ Values of Parameters } \\
\cline { 2 - 5 } \multicolumn{1}{c}{$a_{i}$} & $b_{i}$ & $c_{i}$ & $d_{i}$ \\
\hline RegularSpiking $(R S)$ & 0.02 & 0.2 & -65 & 8 \\
Chattering $(C H)$ & 0.02 & 0.2 & -50 & 2 \\
FastSpiking $(F S)$ & 0.1 & 0.2 & -65 & 8 \\
\hline
\end{tabular}

Here, the neuron index is denoted by $i, j=1,2, \ldots, N$ and the state of the $i$ th neuron at time $t$ is characterized by two state variables: the membrane potential $v_{i}(t)(\mathrm{mV})$ and the membrane recovery variable $u_{i}(t)$. While $I_{i}^{\text {ext }}$ describes the external applied currents. $I_{i}^{\text {syn }}$ accounts for the total incoming synaptic currents into neuron $i$ from neuron $j$-s. The noise current $D \xi_{i}$ represents the external or intrinsic fluctuations of the neuron itself, where $D$ refers to the intensity of noise, $\xi_{i}$ is a random process without time correction, and it is uniformly distributed on the interval $[-1,1]$. On the basis of equation (3), $V_{\text {peak }}=+30 \mathrm{mV}$ is the maximum membrane potential. Here, these three typical properties of neurons correspond to different values of parameter $a_{i}, b_{i}, c_{i}$ and $d_{i}$ are clearly depicted in Table 1 . The Newman-Watts (NW) small-world network is considered as the underlying structure. We performed the network simulations with $N=1000$ neurons. It is emphasizing that the excitatory neurons contain two different types of neurons, which are $N_{R S}$ and $N_{C H}$, respectively, satisfying the condition $N e=N_{R S}+N_{C H}=800$. While the FS neurons are used to imitate the inhibitory neurons, and the number of FS neurons is $N i=N_{F S}=200$, as shown in Fig. 1.

Moreover, an improved method presented in (Golomb and Rinzel 1993; 1994) is adopted as a global parameter to measure the synchronization degree of neural network.

\section{Experimental Results and Conclusions}

To study the synchronization and gamma oscillation transition, the number of $\mathrm{CH}$ neurons, the weights of synaptic connections, the connection probability and the number of nearest neighbors of each node in the small-network were chosen as the control parameters. Figure 2 shows the evolution of the synchronization degree $S$ and the percentage of $\gamma$ band rhythm versus different parameters. Moreover, the synchronization degree $S$ as a function of the number of $\mathrm{CH}$ neurons and different key parameters are plotted in Fig. 3. Through the experiments, the network becomes more and more synchronous, and then reaches a stable level by increasing the coupling strength, the connection probability, and the number of the nearest neighbor of the network. Besides, the neural network with chattering neurons can arrive synchronization easier than the fast spiking neurons, and it is more susceptible to all the parameters. What is significant is that the synchronous behaviors are in accordance with the gamma oscillations. In addition, our results might give significant implications to better understanding the information processing, especially ameliorating the performance of SNNs in artificial intelligence.

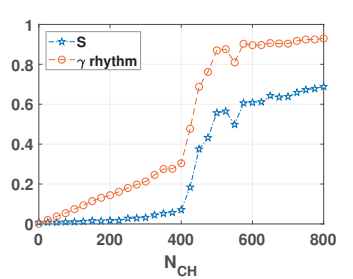

(a)

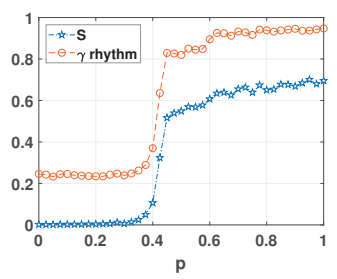

(c)

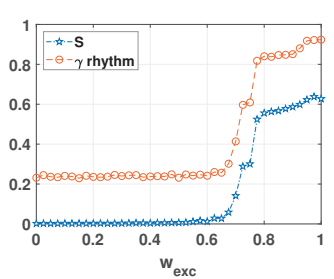

(b)

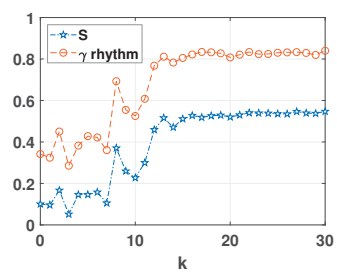

(d)
Figure 2: The evolution of $S$ and the percentage of $\gamma$ band rhythm versus different parameters. (a) $N_{C H}$, (b) $w_{\text {exc }}$, (c) $p$, and (d) $k$.
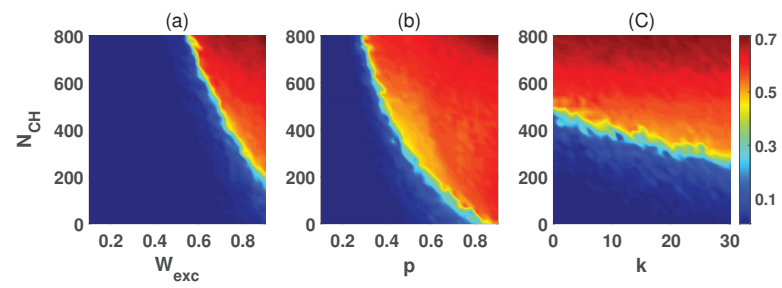

Figure 3: (Color online) $S$ as a function of $N_{C H}$ and different key parameters. (a) $S$ versus $w_{\text {exc }}$ and $N_{C H}$, (b) $S$ versus $p$ and $N_{C H}$, and (c) $S$ versus $k$ and $N_{C H}$.

\section{Acknowledgments}

This work was supported by the National Science Foundation of China (No. 11772069) and the National Key Research and Development Program of China (No. 2018YFB1003804).

\section{References}

Golomb, D., and Rinzel, J. 1993. Dynamics of globally coupled inhibitory neurons with heterogeneity. Physical review E 48(6):4810.

Golomb, D., and Rinzel, J. 1994. Clustering in globally coupled inhibitory neurons. Physica D: Nonlinear Phenomena 72(3):259_ 282.

Hassabis, D.; Kumaran, D.; Summerfield, C.; and Botvinick, M. 2017. Neuroscience-inspired artificial intelligence. Neuron 95(2):245-258.

Izhikevich, E. M. 2003. Simple model of spiking neurons. IEEE Transactions on neural networks 14(6):1569-1572.

Wu, Y.; Deng, L.; Li, G.; Zhu, J.; Xie, Y.; and Shi, L. 2019. Direct training for spiking neural networks: Faster, larger, better. In Proceedings of the AAAI Conference on Artificial Intelligence, volume 33, 1311-1318. 